\title{
A Survey on Multi Objective Optimization Challenges in Swarm Intelligence
}

\author{
${ }^{1}$ Rich Caruana and ${ }^{2}$ Yin Lou \\ ${ }^{1,2}$ School of Computing, The University of Utah, UT 84112, United States. \\ ${ }^{1}$ caruanamimma@hotmail.com
}

\author{
Article Info \\ Journal of Computing and Natural Science (http://anapub.co.ke/journals/jens/jcns.html) \\ Doi: https://doi.org/10.53759/181X/JCNS202101018 \\ Received 20 March 2021; Revised form 08 May 2021; Accepted 28 June 2021. \\ Available online 05 October 2021. \\ (C)2021 Published by AnaPub Publications.
}

\begin{abstract}
Various challenges in real life are multi-objective and conflicting (i.e., alter concurrent optimization). This implies that a single objective is optimized based on another's cost. The Multi-Objective Optimization (MOO) issues are challenging but potentially realistic, and due to their wide-range application, optimization challenges have widely been analyzed by research with distinct scholarly bases. Resultantly, this has yielded distinct approaches for mitigating these challenges. There is a wide-range literature concerning the approaches used to handle MOO challenges. It is important to keep in mind that each technique has its pros and limitations, and there is no optimum alternative for cure searchers in a typical scenario. The MOO challenges can be identified in various segments e.g., path optimization, airplane design, automobile design and finance, among others. This contribution presents a survey of prevailing MOO challenges and swarm intelligence approaches to mitigate these challenges. The main purpose of this contribution is to present a basis of understanding on $\mathrm{MOO}$ challenges.
\end{abstract}

Keywords - Multi-Objective Optimization (MOO), Particle Swarm Optimization (PSO), Pareto Optimal (PO), Traveling Salesperson (TSP), Ant Colony Optimization (ACO).

\section{INTRODUCTION}

Due to complexity of Multi-Objective Optimization (MOO) problems [1], no one solution can meet all of the goals at once. There are opposing objectives in this situation. There can be no improvement in any of the optimal solutions without worsening the value of the other objective functions, in which case a solution is said to be nondominated, Pareto optimum, Pareto efficient, or noninferior. There may be a (infinite) number of Pareto Optimum (PO) solutions that are all judged to be equally desirable if there is no extra subjective preference information. Delhoum [2] approach MOO issues from a variety of angles, resulting in a wide range of approaches to problem formulation and solution. Discovering a definitive answer that fulfills a decision maker's subjective preferences while also finding a representative collection of Pareto optimum options are all possible goals.

Multi-Objective Optimization (MOO) issues may be approached in one of two ways. (i) The Traditional Method [preference-based MOO] (ii) The Best Strategy [Pareto Optimal (PO) strategy]. Solving a multi-objective optimum problem is as simple as combining the objectives into a synthesized objective variable whose value is proportionate to the choice vector supplied to each unique target. The MOO issue is reduced to a single-objective optimization issue using this approach of scalarizing a goal vector into a single aggregate decision variable. Most of the time, when a compound optimization algorithm is maximized, only one trade-off solution is viable. This method for dealing with MOO issues is straightforward but rather subjective. This is a preferences-based MOO method. Finding a complete collection of nondominated alternatives is the second technique. It is referred to as the PO set.

When moving from one Pareto approach [3] to another, one or more objectives must be relinquished in order to achieve a certain level of gain in others. When it comes to solving real-world problems, PO approach sets are often preferred over single remedies. The magnitude of the Pareto established frequently expands as the number of goals increases. The comparative priority vector used to structure the reinforced function has a significant impact on the results of a preferencebased strategy. It is possible to get a (ideally) distinct trade-off remedy upon the modification of this preferential vector. Every scenario does not require preferential vectors to guide to a trade-off optimal solution. It's also worth mentioning that comparative preferential vectors are highly contextual and problematic to obtain. On the other hand, the best MOO technique is impartial. The primary purpose of this is to create as many diverse trade-off options as feasible. A deeper knowledge of the issue is needed to choose a single cure from a collection of trade-off possibilities that have been uncovered so far.

It is necessary to supply a vector of proportional preferences in the conventional technique, but in the proficient method, issue information is utilized to pick one of many trade-off alternatives for remedy selection. A more practical and less emotive technique is better in this scenario. To achieve the rationale of this research, this paper has been organized as follows: Section II presents the scope and objective of the research. Section III presents a critical survey of the research. Lastly, Section IV concludes the paper. 


\section{SCOPE AND OBJECTIVE}

The ideal values or the optimal idea may be obtained via the optimization process. Maximizing or minimizing one's goal is one of the most common optimization tasks. This kind of optimization is called multi-objective optimization (MOO). Problems like this one may be encountered in a wide range of fields - from science and mathematics to sociological research and economy. Optimizing fishery bioeconomic models may benefit from MOO's economic application. This model may be used to provide the best possible estimates of resource extraction and control plan performance. This model is based on the principle of open access economics, or communal domain, which is developed from the demographic increase logistic paradigm. It is the author's goal to build a framework for the North Sea fishery with four goals in mind: maximize revenues, preserve quota sharing across nations, keep employment in the sector, and minimize wasted resources.

Identifying who will profit from a political campaign is the goal of using MOO in governance. The Eigenvector central averages, the proximity between significant participants, and two routers' topologies, the Dolphin Networks and the Prisoners Network, were all examined in this research. By presuming the chosen important player must operate well individually or as a whole, determining the greatest major player is accomplished. They may be used for heating and cooling in the area of mechanical engineering. Utilizing the Genetic Algorithm (GA) program, it is employed to reduce the overall cost of technology, encompassing investment capital and yearly energy consumption, comprising pumping. In addition, the height of a heating element is being reduced. Optimizing design factors such outer tube thickness, outside tube length, and baffles width is the focus of MOO.

This paper focusses on a survey of the MOO challenges and the swarm intelligence remedies to it. It is possible to tackle MOO problems in one of two ways. Traditional Method Pareto Optimal (PO) strategy; and the Best Strategy. An easy method for solving multi-objective optimality problems is to combine each goal into a single synthesized objective, whose value corresponds to the choice vectors provided to each target. Scalarizing a goal vector into a single aggregate decision variable reduces the MOO problem to a single-objective optimization problem. Only one trade-off solution can be found when using a compound optimization method to its full potential. However, this approach of resolving MOO concerns is simple but subjective. MOO that is based on personal tastes. The second method is to find a comprehensive array of non-dominant alternatives. Section II presents a critical survey in relation to the research aim and scope.

\section{SURVEY}

\section{Challenges}

The multi-objective optimal control issue necessitates continuous consideration of a number of competing goals. Rather than finding a single optimum alternative, the purpose of MOO is to find a limited number of Pareto optimum alternatives [4]. For instance, a multi-objective issue that concurrently optimizes (without sacrificing generality) goals might be expressed as follows:

Reduce $\left\langle f_{1}(x), f_{2}(x), \ldots f_{1}(x)\right\rangle$ in relation to $X \aleph S$. Where $S$ represents the remedial space that is feasible. To mitigate the multi-objective challenge, fundamental concerns need to be handled with care. One of the concerns is the optimization of distinct objective functionalities at the same time. A critical concept integrated in MOO is domination. The researchers illustrate domination using Def. 1:

Def. 1: The remedy $x^{1}$ is considered to dominate another remedy $x^{2}$ incase all the condition (i) and (ii) are true. (i) the remedy $x^{1}$ is no worse compared to $x^{2}$ in the various objectives, or $f_{j}\left(x^{1}\right) \neq f_{j}\left(x^{2}\right)$ for $j$ is $1,2 \ldots . \mathrm{M}$; and (ii) the remedy $x^{1}$ is better compared to $x^{2}$ whereby at least a single objective, or $f_{j}\left(x^{1}\right) \neq f_{j}\left(x^{2}\right)$ for about a single $j$ is $1,2 \ldots$ M.

Non-dominated remedy set is illustrated using Def. 2.

Def. 2: Among the remedy set $\mathrm{P}$, non-dominated remedy set $\mathrm{P}$ ' include the ones not dominated by any set $\mathrm{P}$ members.

The Pareto optimum set of alternatives is a collection of non-dominated options. For the resolution seeker, this set contains all of the available excellent remedies. MOO issues have been addressed using a variety of methodologies, including Evolutionary Algorithms (EAs), Ant Colony Optimization (ACO), and Particle Swarm Optimization (PSO), which are discussed in this article. PSO is well-known for its ability to solve a variety of static issues. It has been hypothesized that EAs might do well in dynamic settings, which are common in the real world. PSO approaches, on the other hand, have promise for dynamic situations. Ant Colony Management is also another MOO method that is gaining prominence. Classic issues like as assigned difficulties, sequencing and scheduling challenges, chart colouring, discontinuous and continuous function enhancement, and route optimization issues are just a few of the disciplines where ACO has been used and shown to operate effectively. Cell arrangement difficulties in embedded systems, communications system architecture, and bioinformatics are some of the more current uses.

\section{Remedies}

\section{Evolutionary Algorithms (EAs)}

Multi-Objective Optimization (MOO) issues are generally well-suited to Evolutionary Algorithms (EAs). EAs have been employed in a variety of applications, including autopilot engineering, vehicle scheduling, and the Traveling Salesperson (TSP) issue. Mittal et al. [5] discussed some conceptual elements of evolutionary MOO. The research on evolutionary computation for the vehicle navigation issue, a MOO issue, was studied by the researchers. According to the researchers, 
real-world applications have multiple competing goals. The authors established basic principles of MOO highlighting the reason and benefits of employing evolutionary methods.

Multi-objective issues, according to Li et al. [6], are often difficult, requiring the adjustment of a large variety of factors as well as the optimization of numerous goals. MOEAs (multi-objective evolutionary methods) are a prominent method of dealing with MOO issues by using evolutionary research methods. Due to these issues, EAs have become the preferred tool. EAs that can retain a set of solutions may also investigate many regions of the Pareto frontier at the same time. Basic EA function is shown in the flow diagram in Fig. 1. The Pareto optimum set was discovered using a biological approach in [7].

[8] explains the issue characteristics that may make it challenging for a multi-objective evolutionary algorithm to reach the genuine Pareto-optimal front. He created multi-objective test cases with MOO-specific characteristics, allowing academics to evaluate their algorithms for certain areas of MOO. From a technical aspect, the researchers looked at the efficacy of basic tripartite GAs on a variety of easy to difficult test scenarios. Even though the crossovers regulator may also be used to solve similar issues, crossover operators play a significant role in addressing basic issues. When these two processors are used separately, they provide two distinct overall population operating zones. The mutation operation is the main search operator for difficult issues requiring enormous multimodality and deceit, and it works dependably with a large enough size of the population.

If the population density is sufficient, they proposed using the genetic operators. By way of introduction, [9] provided the Pareto Envelope-based Selection Algorithm (PESA). The authors analyzed four multi-objective EAs statistically. Stability Pareto Evolutionary Algorithms (SPEA) is an evolutionary technique to multi-criteria assessment that incorporates several aspects of prior EAs in a unique manner to provide greater results. SPEA was also developed by the researchers. To deal with MOO scenarios that include both linear and non-linear limitations, the authors developed a MultiOBjective Evolution Strategy (MOBES). A set of PO alternatives is generated in each iteration using the technique they suggest.

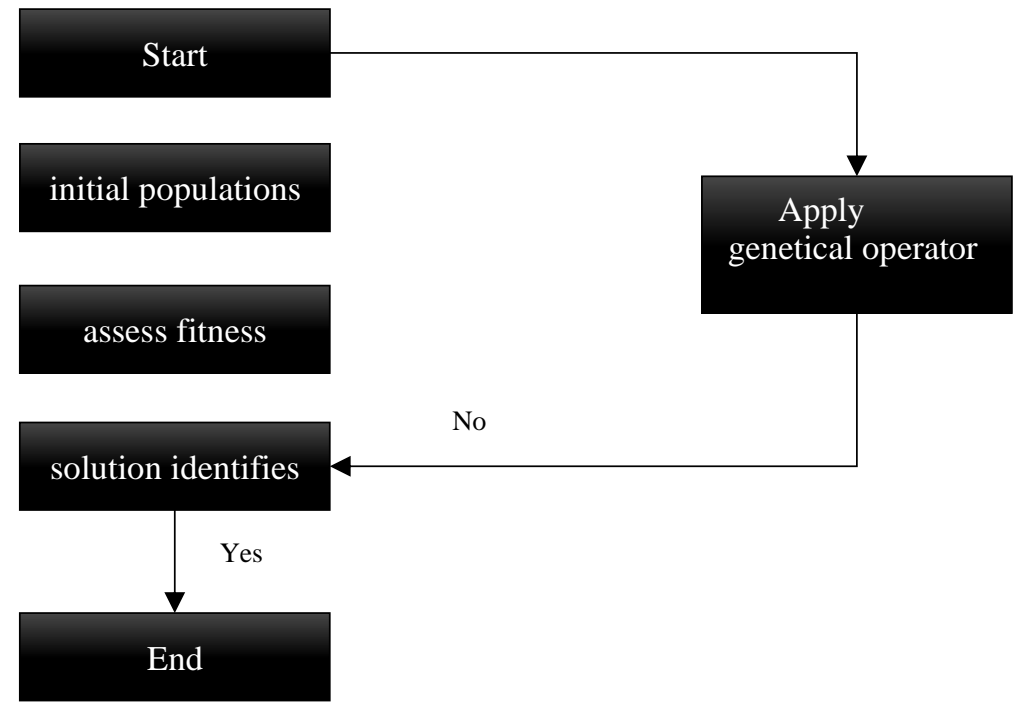

Fig 1. Evolutionary Algorithms (EAs) flowchart

Some researchers tweaked genetic techniques to improve the solutions to the challenges they were testing. Yin and Li [10] used a Modified Genetic Particle Swarm Optimization (MGPSO) to address constrained vehicle routing issues in this set of studies. He combined PSO with the mechanics of genetic replication (crossover and mutation). A numerical coding and decoding format are used in this approach. Five well-known CVRP standards have been used to test this approach. Prins devised a hybrid GA to handle the car routing issue that is both simple and efficient.

Mesquita-Cunha, Figueira, and Barbosa-Póvoa [11] proposed that the population's weights are consistently changed over mutation, leading to the Pareto frontline. They looked at two methodologies: assigning a consistently dispersed random quantity to each person in the society in each iteration, and assigning a uniformly decentralized arbitrary weight to each member from the group in each century. The alternative option is to modify the weight on a regular basis as the procedure evolves. They discovered that the community may embrace the Pareto frontier in both circumstances, however not all of the observed Pareto alternatives are retained in the community. The Stability Pareto Evolutionary Algorithms (SPEAs), Pareto Converging Genetic Algorithms (PCGAs), and Non-dominated Sorting Evolutionary Algorithms (NSGAs).

\section{Particle Swarm Techniques}

Particle Swarm Optimization (PSO)

The little birds fly in unison and exhibit great synchronicity in turning, taking off, and landings, despite the absence of an apparent leader. They hypothesized that synchronisation of movements may be a result of each bird in the flocks following 
"guidelines" for movement. The notion of PSO was developed using a modeling of a bird swarm. PSO was created by simulating bird swarming in two-dimensional areas. PSO's research techniques are as shown in:

- At each location it encounters in reality, each particle assesses the functional in order to optimize it.

- Each particle keeps track of the best value it's discovered so far (pbest) as well as its dimensions.

- Each particle is sensitive to the global best location that one of the flock members has discovered, as well as its global best deal (gbest).

- $\quad$ Each particle estimates its new acceleration using the dimensions of pbest and gbest.

The basic PSO algorithms is given as:

(i) generate the first swarm

(ii) repeat for every particle (i) does

a. $\quad$ In case $f\left(X_{i}\right) f\left(P_{i}\right)$ then $P_{i} \leftarrow X_{i}$

b. $\quad P_{i}=$ maximum $\left[P_{\text {neighbors }}\right]$

c. Update speed

d. Update position

e. end for:

(iii) until termination criterion is met

The Discrete Binary PSO method was presented by Queen and Auxillia [12]. The Hamming distance between the particle at time $t$ and time $t+l$ is used to represent the particle's velocity in this procedure. discrete space vid was described as the chance of getting the value 1 . The source of this information could not be located. The discrete version remains same except that pid and xid are now discrete. Since Vid is a probability, it must fall within the range of 0 to 1 .

The resultant positional transition is therefore determined using:

If $\left[\right.$ rand ()$\left.<S V_{d}\right]$

Therefore $X_{d}=1$

Otherwise $X_{d}=0$

Whereby $S V_{d}$ represents a sigmoid that limits transformations and rand () represents the quasi-random numbers chosen from uniform distributions in $[0,1]$.

They found that binary particle swarm design is capable of tackling a wide range of issues quickly and efficiently based on their findings. It has also been shown to be possible to optimize any continuous or discrete function using a discrete version of the method, which enhances its possibilities. EAs and the notion of PSO have been combined in a novel way by researchers. This model was evaluated and compared to the standard PSO and the standard GA in terms of performance. PSO with Gaussian mutation outperformed regular GA and PSO in the trials on unimodal and multimodal functions. Binary PSO was first proposed by Aygun et al. [13].

\section{Neighborhood Topologies}

The particle swarm method is characterized as a collection of particles whose paths fluctuate around a zone specified by each particle's prior greatest performance and the achievement of another particle. To find "some other particle" to impact the person, several ways have been utilized. The particles in the initial PSO method were impacted by their own location as well as the positioning of the component that determined the optimal result for the value. They combined gbest and pbest structures to increase the efficiency of basic PSO and evaluated the method on six typical benchmark measures while focusing on two aspects: number of neighbours and amount of classifying. The findings showed that the Von-Neumann topologies (with or without self) performed well, whereas star and gbest (without self) performed poorly. Various geometrical configurations are shown in Fig. 2.

The standard particles swarm structure gbest regards the whole community as the person's neighbourhood, with all particles linked directly to the majority's ideal option. In pbest type statistical manner, on the other hand, a person's residence is made up of the demographic array's neighboring members. It is the sluggishness and most deceptive mode of communicating. The gbest type converges swiftly on issue remedies but is prone to become caught in local optimization, while pbest individuals may move around global optimum as subgroups investigate various areas. They observed that when node ranges are too low and interaction is too fast, the population rapidly converges on the optimal solution identified in the early development stages. In this circumstance, the community will be unable to go beyond the zones that are locally optimum.

Restricting communications too much, and from the other hand, leads in ineffective trial allocations, as single particles roam about aimlessly in the search area. Von Neumann sociometry outperformed the conventional ones on a set of standard testing issues, hence it was selected out of the surrounding setups they evaluated. New techniques for an individual element to be impacted by its surroundings were developed by Rachdi et al [14]. Various topological structures have been shown in Fig. 2. 


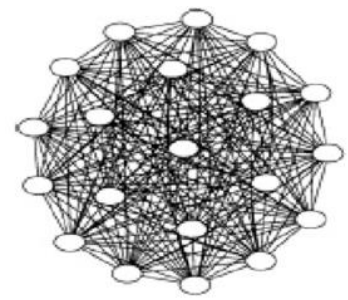

Global best (all)

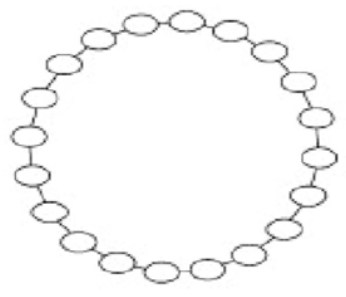

Ring

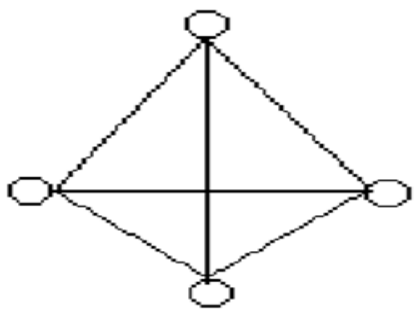

Pyramid

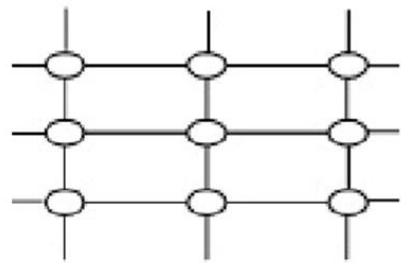

Square

Fig 2. Configurations of neighborhood topologies

Gamorez, Nianga, and Canoy [15] created and tested numerous sociometries, including the gbest, pbest, four groups, VonNeumann, and pyramidal framed triangle, as reported by them. They employed two efficiency metrics: (i) The best functional outcome following particular number of times; and (ii) The most efficient results following a particular iteration number; the number of points the system required to fulfill the criterion, with five typical test values used. They discovered that not only is the person impacted by the greatest person in the area, but that the best outcomes also disregard the person's experiences. They came to the conclusion that von-Neumann sociometry is a strong performer, but they did not explain why. The researchers developed a constantly changing ring design in which elements are connected in a transversal manner to their own optimal utility. In the meantime, two tactics are utilized to allow specific elements to interact with their neighbors: the "Learn from Far and Nicer Ones' ' approach and the "Centroid of Mass' ' approach. The suggested system's efficacy is supported by empirical findings on six benchmark measures. By analyzing concerns like inter-particle interactions and chances of selections depending on the particle rankings, the researchers made the first step towards that analysis method of the pbest PSO with changeable random neighborhood topologies. In order to achieve better results, the researchers combined star, ring, and Von-Neumann designs. The particle's mobility will be computed independently for each topology, and its velocity will be updated according to the configuration that is better suited. As a result, the particles will choose the most advantageous option for themselves. In comparison to previous techniques, this approach, according to the researcher, performed best on nine typical test measures and had a quicker convergence time.

\section{Dynamic Environments}

Most implementations are dynamical, necessitating an optimization technique with the ability to follow the goal's fluctuating location. A strategy for modifying the PSO for dynamic situations was developed by the researchers. As the conditions change, the procedure causes each component to refresh its database of its optimum location in order to prevent making directions and acceleration judgments based on obsolete knowledge. The following are two approaches to starting these procedures:

a) Triggered restarting, depending on the number of environmental changes, and

b) Periodic resetting, depending on the iterations count.

The two improvements enable PSO to search both in dynamic and static situations. PSO was tweaked by the researchers to autonomously follow alterations in a complex model. On two benchmark operations, Orlando and Ricciardello [16] explored various environmental prevention and detection strategies. They also implemented re-randomization to respond to changes occurring. They also developed an adaptive PSO that continuously monitors numerous changes in a complex system. The authors looked at many PSO versions that function well in dynamic contexts. Their fundamental concept is to divide the particle populations into a series of interaction swarms. An exclusion parameter interacts with these swarms on a local level, while an anti-converging operator interacts with them on a global level. The multi-swarm technique beats earlier methods when tested on multisensory dynamic shifting peaks benchmark parameters. On several dynamic benchmark functions, the authors examined dynamic variations of regular PSO and Hierarchy PSO (H-PSO).

Orlando and Ricciardello also presented the Partitioned H-PSO (PH-PSO), a hierarchical PSO. The PH-PSO technique is modified to split the hierarchy into distinct sub-swarms for less numbers of iterations. In reference to all the tests systems, H-PSO reported better performances compared to PSO, even though PH-PSO is reported to have performed better than PSO when it came to determining differences with small tweaks. Later work on noisy and turbulent conditions examined the ACO technique for dynamic and noisy function approximation. For a given number of generations after environmental changes, PH-PSO had its molecule's hierarchy maintained into distinct sub-swarm. Metaheuristics often cope with noise by using functional reevaluations. The hierarchy was used to select a subset of items for which possibly re-evaluations are fundamental to minimize the re-evaluation numbers that are needed. They also demonstrated how to use a strategy to discover changes in the optimization method in noisy situations.

\section{PSO Algorithm for MOO Problems}

Segmentation Analyses, MOO Algorithms, Artificial Neural Learning, and other disciplines use ACO methods. In this part, we'll look at how to use PSO to solve MOO issues. The first research of the Particles Swarm Optimisation approach 
in MOO Challenges was published by Nugroho, Faisal, and Hunain [17]. They investigated PSO's capacity to discover Pareto Ideal locations and record the Pareto Fronts form. Fixed or adjustable weights may be used in the Generalized Aggregation technique. A multi-swarm PSO that can tackle multi-objective challenges was also created utilizing the Genetic Approaches for MOO. The researchers used a dynamic community ACO strategy to address MOO concerns. For a faster processing time, they offered an extended store to integrate several solutions of Pareto optimal in a single place.

In order to enhance MOO, the researchers created Non-dominated Sorting Particle Swarm Optimizer (NSPSO). For effective non-domination assessments, NSPSO utilizes the personal records and offspring of particles to improve upon the basic version of PSO A particle's individual best and its offspring are examined separately by NSPSO rather than a single study of all of the particles' individual bests. Swarm composition may be accelerated toward a Pareto optimum frontline by applying the correct selection forces using this strategy. The results and comparisons with NSGA II show that NSPSO is highly similar with existing evolution and PSO multi-objective techniques applying the non-dominated grouping paradigm and two parameter-free clustering methodologies.

Pareto dominance was included into PSO as a way to handle situations when there are several goal values. Other components employ a secondary particle depository to direct their own flight as part of this novel strategy. They also included a mutation operation to enhance the system's exploratory abilities. Concurrent variants of the Vector Evaluated PSO (VEPSO) algorithm for multi-objective scenarios have been evaluated on frequently used test cases. Compared to the findings of the Vector Evaluated Genetic Algorithm technique, the acquired results show that VEPSO is more effective. Homogeneous Particle Swarm Optimizer (HPSO) for MOO algorithms has been developed by many scientists. A single global repository notion was offered, which meant that each component had lost its own identity and was simply considered as part of a social group. This PO set was discovered after a series of MOO problems were investigated and tested.

There have been developments in multi-objective PSO systems. There are two types of programs that were categorized: (a) processes that employ each objective function independently, and (b) Pareto-based techniques. Listed in reverse chronological order, the methods for each grouping are described in detail. Academics believe that for MOO difficulties, a large number of uniformly distributed and representative PO solutions is essential. Swarm populations may be accelerated toward the Pareto-optimal frontline by contests and clonal selecting operators, according to the AER (Agent-EnvironmentRules) paradigm, which is based on competition and clonal selection.

According to the qualitative and quantitative correlations, the suggested technique is very effective and may be regarded as a real option for solving multi-objective objective issues. A method for multi-objective PSO was developed by the researchers. The Fuzzy-Pareto-Dominance (FPD) relationship underpins the method. FPD is a general rating technique that maps rating numbers to set component dimensions. These rating values are obtained instantly from the set's component matrices and may be used to execute rank procedures (such as picking the "biggest") on the vectors inside the group. A model or metaheuristic for formally extending single-objective optimization approaches to MOO methodologies, FPD may be considered after such vector sets are constructed. In the case of the Standard EAs, this has been shown in the past. PSO, in which a swarm of particles is maintained, makes use of this concept. A simple optimisation problem shows that the new PSO technique can handle a greater number of objectives and has attributes equivalent to the original single-objective PSO approach (Pareto-Box-Problem). Fuzzy dominance, a numerical metric, was used to develop a hybrid PSO-Nelder Mead Simplex MOO approach. K-means is used to divide the population into tiny units, while PSO is used to change the position and velocity of each particle in every phase. For extra local search, the Nelder Mead simplex method is utilized individually inside each group. On a variety of test scenarios, the method they developed outperformed MOPSO.

Hassan et al. [18] suggested a multi-objective PSO system based on dynamic sub-swarms. It divides particles into multi-sub swarms, each of which uses an enhanced cluster archive approach, and performs PSO in a comparable independent manner, depending on the solutions dispersion of MOO algorithms. Clustering improves the dispersion quality of options in the long run. The choosing demand is increased by selecting the nearest particle from the archived group to the gbest and by developing the pbest choose process. Meanwhile, the dynamical set particle inertia weight, which is proportional to the number of dominant elements, successfully maintains a balance among global and regional discovery in the initial stages. Experiments indicate that, particularly for issues with non-continuous Pareto-optimal frontiers, this method produces high resolution and a great ability to preserve the dispersion of alternatives. To cope with MOO issues, the researchers presented a PSO strategy depending on the intensity Pareto method. To handle distinct MOO issues, the suggested enhanced PSO algorithm is applied in the development of three hybrid evolutionary algorithm PSO (EA-PSO approaches. The differentiated the results to SPEA (Strength Pareto Evolutionary Algorithm) and the competition multiobjective particle swarm optimization utilizing numerous measures, employing seven benchmarks from the research. In comparison to the previous techniques, the suggested approach has a slower convergence but uses less CPU time. PSO and evolution methods are combined to create better hybrid strategies that beat SPEA, the competition multi-objective particle swarm optimization, and the recommend stronger Pareto particle swarm optimization on several criteria. The researchers looked at several approaches to dealing with boundary restrictions and guiding guide selections. The authors also looked at combining many iterations of the program to get higher-quality results. To cover the PO front, the researchers suggested a technique that uses multi-objective PSO. There are two stages to this procedure. The purpose of phase 1 is to approximate the Pareto-front as closely as possible. Sub swarms are formed for the Pareto front in a second iteration.

Zhang et al. [19] describe a general technique for multi-objective optimal designs of complex laminates elements based on the Quantum behaved PSO (QPSO) framework, which employs a unique MOO technique. QPSO is a co-variant of the well-known PSO algorithm, and it has been effectively designed and used for multi-objective composite structure 
optimization. The issue is phrased with many goals in mind, including reducing the weight and overall cost of the composite element while maintaining a defined power. To handle MOO issues, researchers suggested using PSO methods. Their method is focused on the Pareto principle and a cutting-edge 'parallel' computer methodology that aims to increase algorithm efficiency and effectiveness at the same time. The findings show that the suggested method outperforms previous MOEAs in virtually every scenario evaluated, correctly, consistently, and consistently approximating the genuine Pareto frontline.

Durillo et al. [20] presented a Multi-Objective Particle Swarm Optimizer depending on Pareto domination and the usage of a crowded component to filter out the accessible leaders' listing. They also suggested using several mutation processors that work on various swarm subgroups. In addition, the method uses the e-dominance notion to limit the number of obtained results generated by the program. The findings show that the suggested method is very competitive, capable of approximating its front even when all other PSO-based methods fail.

\section{Hybrid Particle Swarm Algorithm}

PSO was combined with a variety of evolving and fuzzy notions by experts. [21], for example, claims that the PSO method suffers from early convergence when solving multidimensional issues with large parameters. The early convergence of the particle swarm paradigm is mostly caused by a drop in velocity profile in the search process, which leads to complete imploding and, eventually, fitness paralysis of the swarm. To get around the issue of immobility, they added volatility to the PSO method. To regulate the velocity of electrons, the method utilizes a minimum frequency criterion. A probabilistic reasoning controller implanted in the Fuzzy Adaptive Turbulent Particle Swarm Optimization (FATPSO) system, tunes the variable, minimum motion threshold of the projectiles, iteratively. The findings of the studies show that the FATPSO beats both SPSO and GA in terms of preventing convergence rate. PSO using EAs is a hybrid technique proposed by the researchers. They suggested utilizing GA to modify PSO. The suggested hybrid systems outperform the regular PSO in tests, according to the findings. Breeding Swarms, a new hybrid GA/PSO technique suggested by the researchers, combines the advantages of PSO and genetic programming. The hybrid method incorporates the PSO velocity profile in addition to the usual velocity and position update rules of Crossover (VPAC). The VPAC crossover activator aggressively disperses the community, stopping it from converging too soon. The hybrid method outperforms both GA and PSO in contrast to the classic GA and PSO algorithms.

\section{Ant Colony Optimization (ACO) Techniques}

The Ant System (AS) can solve issues including the travelling salesperson issue, quadratic assignments, and job planning. Taking its cues from the foraging behavior of real ants, we developed ACO. Ants begin their search for food by randomly scouting the area surrounding their nest. Ants return some of their food to the nest when they find a food source that they think is adequate in quantity and quality. Upon returning to its nest, the ant creates a biochemical pheromone trail in the ground. The amount of pheromone generated by other ants will lead them to the food source, which may vary based on the amount and quality of the food. Stigmergy, or communications via the surroundings, is the key mechanism behind such relationships. Pheromone depositing on routes accompanied by ants is one instance. Pheromone is a powerful hormone that ants can detect as they walk along paths. Because it fascinates ants, they prefer to follow routes with high pheromone levels. This results in an autocatalytic action, in which the process accelerates on its own. Ants fascinated to the pheromone will leave more of the same from the same path, attracting even more ants.

Swarm intelligence is particularly appealing for transferring systems, robotics, and optimization because of this feature. The original ant method is suggested to be extended in a variety of ways. These methods outperformed the initial ant method, delivering much superior outcomes. The employees of the Argentine ant (Iridomyrmex humilis) start exploring a chemical unmarked habitat at random.

Other explorers are recruited as the exploratory front advances, and a route leads from it to the nest. Unlike recruiting trails, which are usually built between two places, these exploring paths have no definite goal and closely mimic army ant foraging habits. A basic trail creating and following action by individual employees may form the exploration pattern, demonstrating how sophisticated collective systems in insect's colonies can be built on self-organization. The impact of studying ant behavior on problems resolution and optimisation was investigated by many researchers. They presented a decentralized problem-solving ecosystem and suggested that it be used to solve the TSP challenges. In [22], Chagas and Wagner suggested ACO depending on the ants' foraging behaviour. Positive comments, distributed computing, and the employment of a constructively greedy heuristics are the major features of this paradigm. To tackle the dilemma of the TSP, ACO is used. The authors advocated the creation of an artificial ant colony that may solve the dilemma of the TSP. Using data acquired in the manner of a pheromone track placed on the vertices of the TSP chart, the synthetic colony's ants are capable of producing increasingly smaller viable tours.

The following are the stages in the fundamental algorithm that they invented:
i. Establish the parameters, conduct the initialization of the pheromone trail
ii. where (termination status has not been met) establish
a) Constructs ant remedies
b) Launch local searches
c) Update pheromones
iii. end where 
Experiments show that the artificially ant colony can provide excellent remedies towards both symmetrical and asymmetrical TSP situations. The approach is an illustration of effective use of a naturalistic paradigm to develop an optimization technique, similar to simulation annealed, artificial neural, and evolution computing. The TSP issue was solved using a different algorithm developed by $\mathrm{Wu}$ et al. [23]. A group of collaborating agencies known as ants collaborate in his technique to discover good TSP remedies. Ants collaborate by depositing pheromone on the corners of the TSP chart while constructing remedies, which serves as an implicit medium of interaction. ACS beats other nature-inspired systems like EAs and evolutionary computing, according to the findings. They came to this conclusion by evaluating ACS-3-opt, a variation of ACS with a localized search technique, against some of the highest functioning symmetrical and asymmetrical TSP methods.

The ACO metaheuristic was proposed by the Serra and Venini [24], who provided discontinuous optimization methods inspired by observations of ant colonies exploring behavior. In response to the deployment of a city of Traveling Salesman Challenge cases, the researchers examined ways for pheromone modulation of ant systems. They offered three approaches to pheromone diversity based on the normalization of pheromone levels at the margins. One technique works worldwide, regardless of where the inserted/deleted city is located. The other approaches only modify pheromones in the implanted city's community, which is characterized diversely in each strategy. As the name suggests, this TSP is based on the concept of a "probabilistic traveling salesman." We are looking for an earlier tour with the lowest projected length among all consumers, utilizing the method of re-visiting a random sample of customers in similar fashion to how they appear on the subsequent tour. Numerous heuristic strategies to the car sampling issue were reviewed and compared by the researchers. The TSP was solved using ACO, which was first presented as a decentralized method.

\section{CONCLUSION}

When solving optimization problems involving many objective functions, Multi-Objective Optimization (MOO) (also referred to as vector optimization, multi programming, pareto optimization or multicriteria optimization), is used. There are numerous areas of research where optimum judgments must be made in the context of tradeoffs between multiple competing goals where multi-objective optimization has been used, including engineering, commerce and logistics. Multiobjective optimization issues involving two and three goals, such as reducing cost while optimizing comfort when purchasing an automobile, and maximizing performance while limiting fuel consumption and emissions of pollutants in a vehicle, are examples. There might be more than three goals in real situations. Even though there are numerous strategies in the research for solving Multi-Objective Optimization (MOO) issues, like evolutionary techniques, Particle Swarm Optimization (PSO), Ant Colony Optimization (ACO), and Hybrid methods that combine all of these approaches. It is still unclear which method is ideal for dealing with multi-objective optimization situations. However, it is not yet clear how these factors may be altered to create better outcomes for various sorts of challenges, and how the remedy seeker will configure the variables to obtain the best solution in the shortest possible time.

\section{References}

[1]. H. Gao, L. Jézéquel, E. Cabrol, and B. Vitry, "Multi-objective robust optimization of chassis system with polynomial chaos expansion method," Eng. Optim., vol. 53, no. 9, pp. 1483-1503, 2021.

[2]. Z. S. Delhoum, "Contribution to multi-level multi-objective linear optimization,” J. Inf. Optimiz. Sci., vol. 42, no. 6, pp. 1383-1395, 2021.

[3]. F. Lievens, P. R. Sackett, and W. De Corte, "Weighting admission scores to balance predictiveness-diversity: The Pareto-optimization approach," Med. Educ., no. medu.14606, 2021.

[4]. A. C. Gomes, R. F. Sampaio, G. C. Barroso, and R. P. S. Leao, "Optimum Pareto method for simultaneous placement of manual and remote controlled switch based on MILP model," in 2021 14th IEEE International Conference on Industry Applications (INDUSCON), 2021.

[5]. S. Mittal, D. K. Saxena, K. Deb, and E. D. Goodman, "A learning-based Innovized Progress operator for faster convergence in Evolutionary Multi-objective optimization,” ACM Trans. Evol. Learn. Optim., vol. 2, no. 1, pp. 1-29, 2022.

[6]. G. Li, Y. Liu, G. Zhao, and Y. Zeng, "Reliability-based robust design optimization in consideration of manufacturing tolerance by multiobjective evolutionary optimization with repair algorithm,” Int. J. Comput. Methods, vol. 18, no. 05, p. $2150005,2021$.

[7]. I. Setiawan, "Maqashid shariah's criticism of the Pareto optimum theory," MUQTASID J. Ekon. dan Perbank. Syariah, vol. 11, no. 1, pp. 14 28,2020

[8]. Z. He, K. Zhou, H. Shu, X. Chen, and X. Lyu, "Multi-objective algorithm based on tissue P system for solving tri-objective optimization problems," Evol. Intell., 2021.

[9]. A. A. Mousa and I. M. El_Desoky, "Stability of Pareto optimal allocation of land reclamation by multistage decision-based multipheromone ant colony optimization," Swarm Evol. Comput., vol. 13, pp. 13-21, 2013.

[10]. S. Yin and H. Li, "GSAPSO-MQC:medical image encryption based on genetic simulated annealing particle swarm optimization and modified quantum chaos system,” Evol. Intell., vol. 14, no. 4, pp. 1817-1829, 2021.

[11]. M. Mesquita-Cunha, J. R. Figueira, and A. P. Barbosa-Póvoa, "New $\varepsilon$-constraint methods for multi-objective integer linear programming: a Pareto front representation approach," arXiv [math.OC], 2021.

[12]. A. A. Queen and D. J. Auxillia, "Simplified discrete binary PSO tuned multivariable PID controller for binary distillation column plant," in 2013 International Conference on Circuits, Power and Computing Technologies (ICCPCT), 2013.

[13]. B. Aygun, B. Gunel Kilic, N. Arici, A. Cosar, and B. Tuncsiper, "Application of binary PSO for public cloud resources allocation system of video on demand (VoD) services,” Appl. Soft Comput., vol. 99, no. 106870, p. 106870, 2021.

[14]. E. Rachdi, Y. E. Merabet, Z. Akhtar, and R. Messoussi, "Directional neighborhood topologies based multi-scale quinary pattern for texture classification," IEEE Access, vol. 8, pp. 212233-212246, 2020.

[15]. A. E. Gamorez, C. G. Nianga, and S. Canoy Jr, "Topologies induced by neighborhoods of a graph under some binary operations," Eur. J. Pure Appl. Math., vol. 12, no. 3, pp. 749-755, 2019

[16]. C. Orlando and A. Ricciardello, "Analytic solution of the continuous particle swarm optimization problem," Optim. Lett., vol. 15, no. 6, pp 2005-2015, 2021.

[17]. F. Nugroho, M. Faisal, and F. Hunaini, "Autonomous groups particles swarms optimisation (AGPSO) to optimise the fuzzy membership function in the automatic watering plant case study,” J. Phys. Conf. Ser., vol. 1908, no. 1, p. 012022, 2021. 
[18]. A. K. Hassan, M. Moinuddin, U. M. Al-Saggaf, and M. S. Shaikh, "On the kernel optimization of radial basis function using Nelder mead simplex," Arab. J. Sci. Eng., vol. 43, no. 6, pp. 2805-2816, 2018.

[19]. Y. Zhang et al., "Preliminary research on abnormal brain detection by wavelet-energy and quantum- behaved PSO," Technol. Health Care, vol. 24 Suppl 2, no. s2, pp. S641-9, 2016.

[20]. J. J. Durillo, J. García-Nieto, A. J. Nebro, C. A. C. Coello, F. Luna, and E. Alba, "Multi-objective particle swarm optimizers: An experimental comparison," in Lecture Notes in Computer Science, Berlin, Heidelberg: Springer Berlin Heidelberg, 2009 , pp. 495-509.

[21]. T. Zheng, J. Wang, and Y. Cai, "Parallel hybrid particle swarm algorithm for workshop scheduling based on Spark," Algorithms, vol. 14, no. 9, p. 262, 2021.

[22]. J. B. C. Chagas and M. Wagner, "Efficiently solving the thief orienteering problem with a max-min ant colony optimization approach," Optim. Lett., 2021.

[23]. X. Wu, Z. C. Wang, T. H. Wu, and X. G. Bao, "Solving the Family Traveling Salesperson Problem in the Adleman-Lipton model based on DNA computing,” IEEE Trans. Nanobioscience, vol. PP, pp. 1-1, 2021.

[24]. M. Serra and P. Venini, "On some applications of ant colony optimization metaheuristic to plane truss optimization," Struct. Multidiscipl. Optim., vol. 32, no. 6, pp. 499-506, 2006. 